Equation (15) indicates that the skewed plate has a smaller torsional rigidity than the unskewed plate and in which way the torsional rigidity varies with the angle of skew $\Lambda$.

5. Pure bending of skewed plate. We denote the applied moment by $M$. The following boundary conditions must be satisfied

$$
\begin{array}{rlrl}
x & = \pm l, & c M_{x} & =M, \\
y & = \pm \frac{1}{2} c-x \tan \Lambda ; & M_{n}=0 .
\end{array}
$$

In addition to this we have the condition of vanishing torque, or of vanishing corner forces $P$, which becomes

$$
M_{x y}+M_{n t}=0 .
$$

From equations (16) to (18) we obtain the following expressions for the coefficients $A$, $B$ and $C$ in $w$

$$
A=-\frac{M}{\left(1-\nu^{2}\right) c D}, \quad B=\frac{\nu M}{\left(1-\nu^{2}\right) c D}, \quad C=\frac{M \tan \Lambda}{2(1-\nu) c D} .
$$

The deflection $w$ is now

$$
w=-\frac{M}{2\left(1-\nu^{2}\right) c D}\left[x^{2}-\nu y^{2}-(1+\nu) \tan \Lambda x y\right] .
$$

In terms of the chordwise variable $\eta$ defined by (12) this becomes

$$
w=-\frac{M}{\left(1-\nu^{2}\right) c D}\left[\left(1+\tan ^{2} \Lambda\right) x^{2}-(1-\nu) \tan \Lambda x \eta-\nu \eta^{2}\right] .
$$

We see from (21) that the skewed plate has a smaller bending stiffness than the unskewed plate and that moreover the applied bending moment produces also a torsional deformation.

\title{
ON THE ERROR TERM IN INTERPOLATION FORMULAS
}

\section{BY IVAN NIVEN (University of Oregon)}

From $n+1$ known values of a function $f(x)$ an approximating polynomial $p(x)$ of degree $n$ can be obtained. If $f(x)$ and its derivatives to order $n+1$ are continuous, then the error term can be calculated from

$$
f(x)-p(x)=\frac{f^{(n+1)}(\xi)}{(n+1) !} \prod_{i=0}^{n}\left(x-x_{i}\right),
$$

where $f\left(x_{i}\right)$ are the known values of the function for $j=0,1, \cdots, n, x$ is an arbitrary point in the interval $\left(x_{0}, x_{n}\right)$ with $x \neq x_{i}$ for every $j$, and $\xi$ is some point in the interval dependent on $x$. The purpose of this note is to indicate that no limit on the error can be obtained by replacing the term $f^{(n+1)}(\xi)$ by some function of an $n$-th difference $\Delta^{n+1} y$. (This replacement is made, for example, in Numerical Mathematical Analysis by J. B. 
Scarborough, 2nd edition, pp. 99-103, the mistake arising from identification of two $\xi$-values in the interval which may be distinct.)

Consider the given data $f(j)=j$ for $j=0,1, \cdots, n$, for which the polynomial approximation is $p(x)=x$. Can the error be bounded by any function of $x_{0}, \cdots, x_{n}$, $y_{0}=f\left(x_{0}\right), \cdots, y_{n}=f\left(x_{n}\right)$ ? That it cannot is clear from the function $f(x)=x+k$ $\sin \pi x$. We have $f\left(\frac{1}{2}\right)=\frac{1}{2}+k, p\left(\frac{1}{2}\right)=\frac{1}{2}$, and $f\left(\frac{1}{2}\right)-p\left(\frac{1}{2}\right)=k$. Since $k$ is independent of $x_{i}$ and $f\left(x_{i}\right)$, this error value cannot be bounded by any function of these without strong hypotheses on the function $f$

A NOTE ON MY PAPER

\title{
ON AXIALLY SYMMETRIC FLOW AND THE METHOD OF GENERALIZED ELECTROSTATICS*
}

\author{
Quarterly of Applied Mathematics, 10, 197-213 (1952) \\ By L. E. PAYNE (University of Maryland)
}

It has been brought to the author's attention that the flow problem for a spindle was considered by E. W. Hobson ("On a class of spherical harmonics of complex degree with application to physical problems", Trans. Camb. Phil. Soc., 14, 211-236 (1889)). Hobson used a method which is entirely different from that employed by the author, but unfortunately his solution is in error. The solution to the problem is given in a corrected and simplified form in this paper. The only reference to Hobson's solution which the author has found is given in the appendix of A. B. Basset's "Treatise on hydrodynamics", vol. 2 (1888). Basset, however, did not discuss the problem and consequently did not recognize the errors in Hobson's solution.

*Received July 21, 1952.

\section{BOOK REVIEWS}

Finite deformation of an elastic solid. By Francis D. Murnaghan. John Wiley \& Sons, Inc., New York, 1951. viii + 140 pp. $\$ 4.00$.

The book contains an unusually lucid exposition of the theory of finite elastic deformations, a field in which the basic physical ideas are often lost in the tangle of complex mathematical notations. The elegance of the present treatment is achieved by the consistent use of matrices. The first chapter serves as an introduction to vectors and matrices. The second chapter is concerned with the strain matrix, its behavior under transformations of the initial and final reference frames, its invariants, and the compatibility relations. The stress matrix and the general relations between stress and strain are discussed in Chapter 4, and for non-isotropic materials in Chapter 5. Chapters 6 and 7 are devoted to the application of the theory to specific problems such as simple shear, simple tension, or torsion of a circular cylinder.

Bringing clarity into a field where confusion is the rule, the volume will doubtless be welcomed by all students of mechanics of continua. In two respects, however, the book disappointed this reviewer. First, there is no reference whatsoever to the considerable volume of classical and recent work in this 